\title{
LAS MUJERES CON DISCAPACIDADES FÍSICAS Y SUS DERECHOS SEXUALES Y REPRODUCTIVOS: IMPLICACIONES PARA LAS POLÍTICAS PÚBLICAS
}

\author{
Yolanda Rodríguez León ${ }^{1}$
}

\section{Resumen}

Este trabajo tiene el propósito de promover el reconocimiento de que las mujeres con discapacidades físicas son sujetos de derechos, específicamente de derechos sexuales y de derechos reproductivos. Además, intereso problematizar el debate sobre la conveniencia e implicaciones de que las voces de las mujeres con discapacidades sean atendidas con políticas públicas y programas que impactan a la población de mujeres en general; o si, por el contrario, debe promoverse la creación de políticas públicas y programas específicos para ellas. El marco conceptual utilizado en este trabajo es el modelo social de la discapacidad, la perspectiva de género y la perspectiva de los derechos humanos.

Los derechos sexuales y los derechos reproductivos de las mujeres con discapacidades físicas han sido ignorados, históricamente. A este sector poblacional no se le reconoce la capacidad ni el derecho de tener una vida sexual activa y placentera; tampoco se le reconoce la maternidad como una opción. A estas mujeres se les concibe como seres inferiores y asexuales, lo que las hace enfrentar el discrimen que promueve y legitimiza las constantes violaciones de derechos que sufren, limitándose así su actividad ciudadana y la posibilidad de ejercer sus derechos. En Puerto Rico, el 20 por ciento de la población

${ }^{1}$ Trabajadora social en la Sociedad de Educación y Rehabilitación de Puerto Rico (SER) y candidata a grado doctoral del Programa de Trabajo Social de la Universidad de Puerto Rico, Recinto de Río Piedras. 
clasificada como femenina tiene alguna discapacidad, pero continúa siendo un sector marginal e invisible para el resto de la ciudadanía y esto incluye a los profesionales y a los hacedores de políticas públicas. Nuestro entorno es uno que asigna rangos de inferioridad al género y a la discapacidad. [Descriptores: discapacidad, género, derechos humanos, derechos sexuales, derechos reproductivos, ciudadanía].

\section{Abstract}

The purpose of this article is to raise awareness about issues affecting disabled women, especially their sexual and reproductive rights. Also, I want to bring to light the issue that women with disabilities have a right to be heard and taken into account regarding political doctrines and programs whose aim is the general female population, or if not, then we must enforce the creation of political aims and programs specific for them. The conceptual frame for this article includes: social model of disability, gender perspective and human rights perspective.

Historically speaking, women with disabilities, sexual and reproductive rights have been ignored. The right or capability to have an active sexual and pleasant life is not recognized neither is maternity an option. These women are often perceived as inferior and asexual. It is because of this prejudice/stereotype that these women are constantly faced with a violation of their rights, therefore limiting their activities as citizens and the possibility of engaging their rights. In Puerto Rico, twenty percent of the female population has a disability; however this sector continues to be marginalized and is basically invisible to the rest of the population, including professionals and public figures. Our environment often assigns inferior ranks regarding gender and disabilities. [Keywords: disability, gender, human rights, sexual rights, reproductive rights, and citizenship].

\section{Introducción}

En el documento de la Federación Internacional de la Planificación de la Familia (IPFF, por sus siglas en inglés, 2008) titulado Derechos Sexuales, se establece que los derechos sexuales y los derechos reproductivos son componentes de los derechos humanos relacionados con la sexualidad y contribuyen a la libertad, igualdad y dignidad de todas las personas. Desafortunadamente, estos son derechos cuyo ejercicio pleno y consciente se les niega a grupos poblacionales socialmente marginados, como son: las personas con discapacidades y de 
una manera más específica, a las mujeres que tienen alguna condición.

La marginación y la opresión son una realidad para las mujeres con discapacidades en el mundo y Puerto Rico no es la excepción. Según el Censo del 2010, el 20 por ciento de la población femenina en Puerto Rico tiene alguna condición o discapacidad. A pesar de ser un sector poblacional significativo, sus voces, intereses y derechos han quedado excluidos de los procesos públicos que impactan sus vidas. Ellas no son consideradas en los asuntos que impactan las vidas de las mujeres sin discapacidades, ni en los asuntos que conciernen a la población con discapacidad, donde prevalecen las posturas masculinas (López, 2007; Morris, 1996; Viñuela, 2009).

Retomando el tema de la sexualidad, es frecuente pensar que las mujeres con discapacidades no tienen interés en las prácticas erótico- afectivas, ni en la maternidad como una opción. Del mismo modo que se presupone que los niños y las niñas no tienen sexualidad, a ellas se les niega la capacidad de sentimiento o expresión sexual y cuando se considera su comportamiento sexual, se hace desde la perspectiva de la desviación (López, 2007).

El presente trabajo tiene el propósito de promover el reconocimiento de que las mujeres con discapacidades físicas son sujetos de derechos, específicamente de derechos sexuales y de derechos reproductivos. Como parte de este escrito, también intereso problematizar el debate identificado en la literatura sobre la conveniencia e implicaciones de que las necesidades e intereses de las mujeres con discapacidades sean atendidas con políticas públicas y programas que impactan a la población de mujeres en general; o si, por el contrario, debe promoverse la creación de políticas públicas y programas específicos para ellas. El marco conceptual utilizado en este trabajo es el modelo social de la discapacidad, la perspectiva de género y la perspectiva de los derechos humanos.

Puerto Rico no cuenta con políticas públicas que atiendan el tema objeto de este escrito. Ya Vélez (2007), había establecido que no se evidenciaba legislación, en los 10 años previos a su investigación, que hiciera referencia directa a las mujeres con impedimentos. En el 2011 realicé, a través de la Legislatura del Estado Libre Asociado de Puerto Rico, una búsqueda de documentos, leyes y medidas legislativas con el 
interés de identificar la existencia de medidas sometidas y/o aprobadas en beneficio de las mujeres con discapacidades físicas en Puerto Rico. La revisión incluyó los años 2001 al 2011 y no se identificó un solo documento que beneficiara a este sector poblacional.

Para subsanar esta ausencia de políticas públicas en Puerto Rico, haré referencia a documentos internacionales que, estableciendo que los derechos sexuales y los derechos reproductivos son derechos humanos relacionados con la sexualidad, deben servir de guía para la creación de políticas públicas en Puerto Rico. España es un ejemplo importante de los países que han asumido la responsabilidad de atender a la población de mujeres con discapacidades físicas a través de políticas públicas. El gobierno de ese país, a través de su Consejo de Ministros, aprobó en diciembre de 2006 el Plan de Acción para las Mujeres con Discapacidad 2007. En la introducción de ese documento se señala que "el Plan de Acción dispone todos los medios necesarios para invertir la tendencia en lo que se refiere al ejercicio de derechos y disfrute de recursos, fomentando su participación y modificando normas y estereotipos discriminatorios" (p.3). Cabe señalar que este Plan de Acción incluye acciones específicas para la atención de los derechos sexuales y los derechos reproductivos de las mujeres con discapacidades físicas. Estas referencias servirán de base a las recomendaciones que realizaré desde la perspectiva del Trabajo Social como disciplina que promueve la justicia social, la integración de los sectores oprimidos y el análisis sistemático de las políticas públicas.

Considero importante señalar que, en este trabajo, al hacer referencia a derechos sexuales y derechos reproductivos aludiré a los consignados en la Carta de Derechos Sexuales y Reproductivos y en la Declaración de Derechos Sexuales, ambos documentos de la IPPF. En el 1995, esta organización defensora de la salud y de los derechos sexuales y reproductivos presentó su Carta de Derechos Sexuales y Reproductivos. Esta carta dio lugar a una mayor conciencia de las diferencias entre los derechos sexuales y los derechos reproductivos. Algunos de los derechos expresados en este documento son: el derecho a la vida plena, a formar una familia, a información y a educación. La Declaración de Derechos Sexuales surge en el 2008 y es un complemento a la Carta. Este documento cuenta con 
diez artículos, entre los que destaco el derecho a la igualdad, a la participación y a la salud. "Esta declaración identifica los derechos sexuales, la autonomía sexual de todas las personas y promueve la salud en un marco libre de discrimen" (Otón, 2010, p. 854).

\section{La construcción de la discapacidad: Una mirada histórica y conceptual}

La discapacidad es un concepto aún en construcción, que ha ido evolucionando a través de la historia. Desde las épocas en las que se utilizaban conceptos como anormales y mongoloides, al hacer referencia a las personas que tenían alguna condición, hasta la actualidad cuando se utilizan con mayor frecuencia los términos personas con impedimentos o discapacidad, se han observado grandes cambios médicos y sociales. Estos últimos fundamentados en reclamos y logros de las personas con discapacidades que, reconociéndose en situaciones de opresión y desventajas, son "las que irrumpen en la historia, las que crean lo nuevo" (Dussel, 1998, p. 495). De esta manera, retan a las hegemonías dominantes que pretenden silenciarlas y subordinarlas negando las diferencias y promoviendo la inequidad.

A través de la historia occidental, las visiones 0 perspectivas sobre la discapacidad han ido cambiando y continúan haciéndolo. Parodi (2002) nos presenta cuatro etapas en la historia de la humanidad que ejemplifican el sentir y obrar para con las personas que tienen discapacidades. Estas son: la etapa del rechazo o exterminio; la etapa de la ridiculización; la época de asistencia caritativa; y la época de intervención educativa y cuidado profesional. Las primeras dos épocas ponen de manifiesto la realidad de abusos, prejuicios y muertes que enfrentaban las personas que nacían con un impedimento físico o mental. En la tercera época, desde mi perspectiva, hay un mayor reconocimiento de la persona como ser humano, pero un ser humano inferior. En la cuarta época (siglos XVIII - XX), los cambios en tratamientos y percepciones que se efectuaron tuvieron mucho que ver con el desarrollo de la medicina, la psiquiatría y el modelo médico de la discapacidad. Modelo que estaré analizando más adelante.

Por su parte, Braddock y Parish (2001), nos dicen que los antiguos griegos y romanos aceptaban la creencia de que las personas con impedimentos congénitos encaraban la ira 
de los dioses y debían morir. Durante la Edad Media se creía que asuntos demoníacos eran el origen de los impedimentos. El Renacimiento influenció el tema de la discapacidad por el surgimiento del método científico. Sin embargo, fue en la época de la llustración, según los autores, cuando se observaron cambios científicos fundamentales que llevaron a los médicos, los educadores y los cuidadores a prestar más atención a las personas con discapacidades. Los mismos autores plantean que "a pesar del aumento en el conocimiento científico y en los servicios para esta población, la sociedad mantenía claros los límites entre la normalidad de unos y la aberración de otros" ( $p$. 13).

El desarrollo histórico de la discapacidad se da como parte de los cambios sociales, políticos, religiosos y económicos que van ocurriendo en occidente. Como hemos visto, se tienen pruebas de la existencia de prejuicios sistemáticos contra las personas con insuficiencias en la sociedad occidental antes del surgimiento del capitalismo. Sin embargo, Oliver (1998) postula que con el desarrollo del capitalismo las personas con discapacidades sufrieron la exclusión económica y social y que, como resultado de esa exclusión, la discapacidad se convirtió en un problema individual que requería tratamiento médico. Se buscaba normalizar a las personas consideradas diferentes para que se ajustaran a una sociedad industrializada que requería y requiere de cuerpos hábiles para la producción. Es en estos postulados que se fundamenta el modelo médico de la discapacidad. Este modelo destaca las deficiencias personales, las categorías médicas y la exclusión social de las personas con discapacidades (Barton, 1998; López, 2007). Desde esta perspectiva es el individuo con discapacidad quien tiene el problema y no la sociedad. Este modelo médico ha sido fundamental en el mantenimiento de las concepciones que la sociedad tiene sobre esta población, así como para la formulación e implementación de políticas y el desarrollo de los servicios que se le prestan.

La historia sigue su curso y el enfoque médico ya no es el único que se considera al analizar la discapacidad. Las investigaciones establecen que para los años de 1960 y 1970 , organizaciones de personas con discapacidades, como la Unión de Personas Físicamente Impedidas contra la Segregación (UPIAS, por sus siglas en inglés) en el Reino Unido, así como las 
investigaciones orientadas desde perspectivas sociopolíticas, promovieron el desarrollo de un nuevo enfoque para atender el tema de la discapacidad. A partir de ese momento, se estableció una importante distinción entre insuficiencia (impedimento) y discapacidad (Anastasiou \& Kauffman, 2011; López, 2007; Muñiz, 2006; Vélez, 2007). La primera, relacionada con las deficiencias (fueran físicas, mentales, señoriales, etc.) y por lo tanto, individuales; y la segunda, tomando en consideración todo el andamiaje social que excluye a las personas con discapacidades de la participación social y de sus derechos ciudadanos. Ese nuevo enfoque se conoce como el modelo social de la discapacidad. Este modelo es uno más progresista, que promueve una visión diferente de la discapacidad pues la define como opresión social y discriminación institucional igual que el sexismo y el racismo (Barton, 1998; Muñiz, 2006; Oliver, 1998). El modelo social brinda un nuevo paradigma para entender la discapacidad reconociendo el carácter social, material y reproductivo de los agentes sociales en interacción. El modelo postula que la discapacidad no es sólo la consecuencia del déficit existente en la persona, sino la resultante de un conjunto de condiciones, actividades y relaciones interpersonales (Disability and Sexuality Briefing Report, s.f.; Vargas Pelayo, 2007).

El modelo social, sin embargo, no toma en consideración aspectos de género. Este modelo está basado en la premisa de que la discapacidad impacta a hombres y a mujeres de la misma manera y en igual grado. En el "Disability and Sexuality Briefing Report" (s.f.) se señala que el "Modelo Social ignora el papel que el género juega en la creación de la doble marginación de las mujeres, especialmente en lo relacionado a los derechos sexuales" (parr. 7).

Es necesario, entonces, incorporar la perspectiva de género en este análisis. Esta perspectiva permite reconocer la desigualdad social y las relaciones jerárquicas de poder entre hombres y mujeres. Además, para Cruz (2004), “....representa una herramienta de análisis útil para entender las construcciones culturales y subjetivas que reproducen el acceso desigual a los derechos sexuales y reproductivos de las mujeres con discapacidades" (p. 150). En esa misma línea, Vélez (2007), plantea que el obviar la diferencia de género no promueve el desarrollo de políticas que aborden el discrimen por género, sobre todo de las mujeres con discapacidades. 
Para atender las necesidades e intereses de estos sectores históricamente marginados, como son las mujeres con discapacidades, se requiere una perspectiva amplia, respetuosa de las diferencias y basada en la igualdad como es la de los derechos humanos. "Esta perspectiva reconoce la intersección entre discapacidad, género, raza, orientación sexual, edad, status social y no privilegia unos sobre otros" (Disability and Sexuality Briefing Report, s.f., parr. 8). Este argumento, con el que coincido plenamente, también fue presentado por Soler y Jaime (2008), quienes plantean que, al analizar la discapacidad y sobre todo el caso de las mujeres, es fundamental que el punto de partida sea el de los derechos humanos. Añaden que en el caso de las mujeres con discapacidades "la defensa de los derechos humanos adquiere la dimensión de género" (p. 6).

El modelo de los derechos humanos incluye la discapacidad dentro de un paradigma de derechos que ha ido surgiendo desde la Declaración Universal de los Derechos Humanos en el 1948. En esta declaración se reconoce que todas las personas tienen derechos civiles, económicos, políticos, sociales, culturales y derechos en desarrollo, (entre los que deben contarse los derechos sexuales y reproductivos), independientemente de las diferencias. Desde esta perspectiva, la discapacidad es reconocida como inherente a la condición humana. Por lo tanto, los documentos internacionales de derechos humanos, sean o no vinculantes (esto es, que obliguen o no a los Estados a cumplir con lo acordado), proveen la plataforma desde la cual los derechos sexuales y los derechos reproductivos de las mujeres con discapacidades físicas pueden ser ejercidos y reclamados para que formen parte de las políticas públicas nacionales.

\section{Discapacidad, género y sexualidad}

Comenzaré esta sección presentando algunos datos estadísticos sobre la población de mujeres con discapacidades en Puerto Rico. Siempre llama mi atención el hecho de que siendo un grupo poblacional numéricamente significativo, continúen estando tan excluidas de los procesos públicos que atañen a las mujeres. Los datos del Censo del 2010, revelan que la población total de Puerto Rico, para dicho año, era de aproximadamente 3, 695,636 habitantes. De las 934,674 personas que reportaron tener impedimentos, 488,669 (52.3\%) son clasificadas como femeninas y 446,014 (47.7\%) son 
clasificadas como masculinas. Además, se reporta que las mujeres con discapacidades logran obtener grados asociados y bachilleratos con más frecuencia que los hombres con discapacidades, pero se les dificulta más obtener un empleo. El tener menos posibilidades de empleo las hace más vulnerables a la pobreza y las aísla de su entorno, limitándose así el desarrollo de relaciones personales y su participación en la vida pública del país.

Las mujeres con discapacidades físicas ${ }^{2}$ son las actrices sociales en este análisis, aún cuando reconozco que las mujeres que enfrentan otros tipos de discapacidades son también víctimas de la opresión y la marginalidad. Seleccioné a las mujeres con discapacidades físicas por entender que ejemplifican de forma muy clara lo que es ser vistas como diferentes. Las muletas, andadores y sillas de ruedas se han convertido en emblemas de la discapacidad y aunque son equipos necesarios para la autonomía y movilidad, también son barreras en las relaciones sociales. López (2007) plantea que esos equipos tienen un significado simbólico que suele ser negativo, que se interpone en las relaciones sociales.

Los cuerpos femeninos también tienen simbolismos relacionados, generalmente, a la belleza, la juventud y la delgadez. Son cuerpos históricamente dominados por el patriarcado, el sexismo y la religión. En el caso de las mujeres con discapacidades físicas, estas viven las mismas opresiones de las demás, pero también las promovidas por la visión que se tiene sobre la discapacidad. Estas mujeres tienen cuerpos diferentes que contrastan con los cuerpos "perfectos" que idealiza nuestra sociedad. Los cuerpos de las mujeres con discapacidades físicas son considerados desviados e inferiores, lo que promueve, a nivel inconsciente, sentimientos y emociones negativas (Esmail, Darry, Walter, \& Knupp, 2010).

En nuestra sociedad las mujeres con discapacidades físicas tienen dificultades especiales para establecer relaciones de parejas y para ejercer prácticas sexuales y reproductivas dado que el aspecto físico es fundamental en estas situaciones. Cruz (2004) plantea lo siguiente sobre este particular:

${ }^{2}$ Al referirme a las mujeres con discapacidades físicas, reconozco que es un grupo heterogéneo y que la discapacidad es sólo una de sus identidades. Además, utilizaré el concepto de discapacidad en sustitución del de impedimento, ya que el primero hace énfasis en la perspectiva social de esta realidad y no sólo en la deficiencia de la persona. 
... así como pertenecer a la categoría mujer va a determinar una serie de condiciones de desigualdad social... también la discapacidad física supone condiciones corporales diferentes que en muchas ocasiones alejan a las mujeres de los estereotipos de belleza establecidos socialmente, lo cual genera otros niveles de desigualdad que obstaculizan sus prácticas sexuales y reproductivas. (p. 157)

Este planteamiento pone al relieve la realidad de que las mujeres con discapacidades físicas, son un grupo poblacional que enfrenta mayores opresiones y estigmatizaciones que sus congéneres sin discapacidades o aquellas cuya condición es menos visible. Tan es así que, para algunas mujeres, dentro de los movimientos feministas, incorporar a las mujeres con discapacidades en sus organizaciones y en sus luchas sería contraproducente pues estas representan la debilidad y la falta de independencia que se desea superar (López, 2007; Morris, 1996). En mi experiencia, líderes feministas en Puerto Rico también han demostrado desconocimiento sobre la realidad que vincula género, feminismo, sexualidad y discapacidad. Como consecuencia, se perpetúan los vacíos en las conceptualizaciones de políticas públicas y programas para las mujeres. "No hay duda de que perduran las visiones sociales de la discapacidad estereotipadas y prejuiciadas" (López, 2007, p. 159).

Dentro de este contexto, las mujeres con discapacidades enfrentan una doble marginación: la de ser mujer y la de tener impedimentos (Morris, 1996; Vargas Pelayo, 2007). Aunque estas mismas autoras reconocen que es necesario cambiar los paradigmas y dejar de verlas como víctimas, la realidad es que la construcción social que existe sobre las mujeres con discapacidades las excluye de actividades y derechos fundamentales, como son: la sexualidad y la maternidad como opción. En el European Disability Forum (2010), se señala que la sociedad no está preparada para que sean madres ya que "esto supone un conflicto directo con la representación social que se tiene de las personas con discapacidad como asexuales, sin roles en la vida y sin un lugar para la reproducción" (p. 3). Sin embargo, existe evidencia de que, consistentemente, las mujeres con discapacidades físicas enfrentan con éxito las visiones $\mathrm{y}$ actitudes hegemónicas que buscan oprimirlas y 
limitarlas en el ejercicio de sus derechos. Específicamente en el área de la sexualidad y la maternidad, las mujeres con discapacidades físicas han asumido actitudes combativas frente al papel de víctimas que se les asigna. Se consideran a sí mismas luchadoras frente a las familias y los profesionales que le niegan el derecho a la sexualidad y a la reproducción (López, 2007; Vélez, 2007). No debemos olvidar que los roles tradicionales, que durante décadas las feministas han rechazado por ser medidas opresoras del sistema patriarcal, en el caso de las mujeres con discapacidades pueden convertirse en afirmaciones sobre su derecho a ser mujeres. Viñuela (2009) amplía este señalamiento indicando que las mujeres con impedimentos han estado confinadas a una categoría distinta, no sexuada y sin acceso a elementos que construyen la categoría patriarcal de mujer (belleza, sexualidad, maternidad). Estas categorías por opresoras que sean desde la perspectiva de las mujeres feministas sin discapacidades, para las mujeres que tienen impedimentos pueden ser algo positivo pues les provee una identidad de género que, tradicionalmente, se les ha negado. Me parece que este es un fenómeno importante a ser analizado desde el feminismo pues permitiría reconocer en las mujeres con discapacidades a esas otras que también sufren opresiones y discrimen; convirtiéndose esto en un punto de encuentro donde se reconozcan las semejanzas y diferencias, y se trabaje en la transformación de las múltiples opresiones que enfrentan.

Un ámbito de la vida de las mujeres con discapacidades físicas que está particularmente marcado por la opresión es el de la sexualidad. La sexualidad es inherente al ser humano y por ende a las personas con discapacidades. El desarrollo pleno de la sexualidad es esencial para el bienestar interpersonal y social; así como un derecho humano (Gil, Rodríguez, \& Soto, 2010). A pesar de esto, y utilizando la perspectiva de Foucault (2002), señalo que el tema continúa siendo silenciado y censurado por los sectores dominantes, entre los que destaco el religioso y el político. La forma en que se maneja el tema de la sexualidad impacta negativamente a la población en general y de una manera particular a las mujeres que tienen discapacidades físicas. Podría decirse que: "el ejercicio de la sexualidad es tomado como privilegio del blanco/a, heterosexual, joven y no discapacitado" (Mogollón, s. f., p. 5). 
Por la forma en que las mujeres con discapacidades han sido concebidas socialmente, no se les orienta sobre cuáles son los servicios de salud sexual y de salud reproductiva, ni sobre la manera de acceder a estos. Esto las priva de poder ejercer sus derechos en el ámbito de la sexualidad. En el estudio de Becker, Stuifberguen y Tinkle (1997), se plantea que estas mujeres enfrentan numerosas barreras para obtener servicios de cuidado de salud reproductiva. Además, tienen que enfrentar la sorpresa y el rechazo de profesionales de la salud que se resisten a la idea de que puedan estar activas sexualmente. La investigación de Nosek y otros (1997), indica que las mujeres con discapacidades carecen de conocimientos básicos sobre su salud sexual y reproductiva, reciben menos atención médica y se enfrentan a oficinas médicas que operan con políticas que las excluyen de sus servicios.

Pronunciarme a favor de las mujeres con discapacidades físicas en un tema tan importante y, a la vez, tan silenciado como es la sexualidad cobra un matiz particular en un país como Puerto Rico donde, además de la doble moral que impera en asuntos relacionados a la sexualidad, la salud no es un derecho fundamental. Según lo expone Villalba (2007), en la ponencia presentada ante la Legislatura de Puerto Rico apoyando la medida legislativa que buscaba elevar a rango constitucional el derecho a la salud (R. Conc. del S. 81), en Puerto Rico, "el Estado no ha asumido el conjunto de obligaciones, establecido en el ámbito del derecho internacional, tendientes a asegurar el acceso igualitario a los servicios de salud, la justa asignación de recursos médicos y tecnológicos, infraestructura y medicamentos" (p. 8). Añado que, en el caso de las mujeres con discapacidades físicas, también tiene que tomarse en consideración la necesidad de eliminar las barreras arquitectónicas, contar con equipo especializado y conocimiento médico dirigido a atender sus necesidades, entre otros. Ante esta realidad, asumo los planteamientos de Otón (2010), cuando establece que "el trabajo de mantener y viabilizar el disfrute y el acceso al ejercicio de los derechos reproductivos y los derechos sexuales debe desarrollarse desde una perspectiva de salud en su más alta concepción" (p. 851). La salud es un derecho fundamental y no sólo una prestación de servicios que puede ser delegada a manos privadas y que puede excluir a sectores socialmente marginados. Puerto Rico necesita 
reenfocarse en el aspecto de la salud, y de forma particular en aspectos de salud sexual y salud reproductiva, de manera que garantice la universalidad, accesibilidad y libre costo de dichos servicios. En este proceso tienen que ser incorporadas las voces tradicionalmente excluidas. Esta participación ciudadana es fundamental para el desarrollo de cualquier sociedad.

\section{Ciudadanía: Requisito para el ejercicio pleno de los derechos sexuales y reproductivos}

En Puerto Rico, la Constitución del Estado Libre Asociado (ELA) se estableció en el 1952 y entre sus principios fundamentales se encuentran democracia, libertad, participación ciudadana, igualdad y justicia. Estos datos son evidencia de que la sociedad puertorriqueña valida los derechos, deberes y dignidad de la ciudadanía que prevalecen aún ante la supremacía del Estado (Montañez, 2010). Se considera importante puntualizar que la Constitución no hace diferencia entre personas. Esto es: la ciudadanía no está definida o categorizada de acuerdo con la habilidad o inhabilidad de los cuerpos, la movilidad o cuanto puedan participar en la economía del país. Este hecho llevó a Vélez (2008), a cuestionar la necesidad de crear la Carta de Derechos de las Personas con Impedimentos en Puerto Rico. Su planteamiento va dirigido a señalar que si la Constitución del ELA tiene una Carta de Derechos que cobija a toda la ciudadanía, ¿por qué una carta especial para la ciudadanía definida con impedimentos? Vélez (2008), señala que el desarrollar leyes específicas para la población con impedimentos puede representar un acto de exclusión pues las mueve de la categoría de ciudadanos a una categoría especial. En esa categoría especial se ubican las personas que son diferentes, aquellas que no compiten en igualdad de condiciones con los estándares sociales hegemónicos, lo que afecta negativamente el ejercicio de los derechos ciudadanos. Dada esta exclusión, a las mujeres con discapacidades físicas se les priva de servicios, de información, de educación y de la toma de decisiones en asuntos que atañen estrictamente a su persona. Sin embargo, continúan identificándose evidencias de que estas mujeres no se mantienen pasivas ante su realidad.

Cruz (2004), como parte de los resultados de un estudio llevado a cabo en México entre el 2000 y el 2002, identificó que las mujeres con discapacidades físicas están conscientes de su situación de opresión y de exclusión por lo que buscan 
transgredir las normas y los estereotipos sociales en interés de ejercer sus derechos sexuales y reproductivos. Las participantes del estudio demostraron tener actitudes combativas y de resistencia, interpretaban críticamente su contexto, buscaban espacios sociales y promovían el establecer relaciones socio afectivas. Esto da muestras del reconocimiento que las propias mujeres con discapacidades físicas hacen de su condición, no de víctimas, sino de ciudadanas con derechos y con interés de ejercerlos.

Retomando el tema de los derechos sexuales y los derechos reproductivos, considero importante hacer un poco de historia. En el documento El estado de la salud sexual y reproductiva en América Latina y el Caribe: Una visión global, Flórez y Soto (2008), establecen que durante los primeros setenta años del siglo XX el énfasis de los países y los organismos internacionales era establecer fuertes programas de planificación familiar dado el descenso en la mortalidad y los altos niveles de fecundidad. En los años ochenta, el énfasis estuvo en la salud materno-infantil procurando reducir la mortalidad de los infantes. Para los años de 1990, se adopta un concepto más amplio de salud reproductiva y derechos reproductivos, incluyendo la planificación familiar y la salud sexual. En la Conferencia Internacional sobre la Población y el Desarrollo de 1994, celebrada en El Cairo, los gobiernos reconocieron y declararon internacionalmente que la salud y los derechos sexuales y reproductivos son derechos humanos fundamentales para el desarrollo. Bajo este nuevo paradigma, un buen estado de salud sexual y reproductiva implica la capacidad de disfrutar de una vida sexual satisfactoria y sin riesgos, así como la posibilidad de ejercer los derechos sexuales y reproductivos. Existen, además, documentos internacionales, como la Convención para Eliminar toda Forma de Discrimen contra la Mujer y la Plataforma de Acción de Beijing, entre otros, que también atendieron el tema de los derechos sexuales y reproductivos, pero no hicieron referencia específica a las mujeres con discapacidades. Esto puede significar el reconocimiento de que estas mujeres tienen iguales derechos que sus congéneres sin discapacidades, como puede ser el resultado de la invisibilización a la que está sometida este sector poblacional. 
Por otro lado, existen documentos internacionales que van dirigidos a atender las necesidades específicas de las personas con discapacidades que incluyen, entre otros asuntos, el de los derechos sexuales y reproductivos. Entre ellos se encuentran las Normas Uniformes sobre la Igualdad de Oportunidades para las Personas con Discapacidad (1993). Este documento, según Sanjosé (2007), es el más importante del siglo $\mathrm{XX}$ relacionado con la discapacidad y los derechos sexuales y reproductivos como derechos humanos. Sin embargo, este documento no incorpora la perspectiva de género. Pocos años después surge el Manifiesto de las Mujeres con Discapacidad en Europa (1997), que fue formulado por un equipo de mujeres con discapacidades. Estas logran vincular la perspectiva de discapacidad y de género. La Declaración de Beijing sobre los derechos de las personas con discapacidad (2000) busca desarrollar estrategias para la equidad y completa participación de las personas con discapacidad, especialmente las mujeres y niñas por su mayor vulnerabilidad. En el 2004, a través del Manifiesto de las Mujeres con Discapacidad, estas exigen que se haga efectiva la recomendación que ya habian expresado en el manifiesto europeo instando a los Estados a garantizarle el derecho a la familia, a las relaciones sexuales y a la maternidad. Incorporan exigencias relacionadas con el acceso a tratamientos de fertilidad, formación adecuada del personal médico que atienda sus embarazos, el desarrollo de programas informativos que atienda los temas de sexualidad, salud sexual y reproductiva dirigidos específicamente a ellas, mayor investigación relacionada con sus situaciones y accesibilidad a los centros de planificación familiar, entre otras. Señalo que no debe pasarse por alto que son las propias mujeres con discapacidades quienes levantando sus voces y organizándose han promovido estos esfuerzos. Desde mi perspectiva, estos documentos son necesarios pues permiten identificar a este sector poblacional sin normalizarlo, más bien incorporando sus subjetividades, a la vez que se incorporan las perspectivas de género y de discapacidad.

Es evidente que ya existen documentos en los que se incorporan la perspectivadegéneroy discapacidad. Sin embargo, en la literatura se mantiene el debate de si las necesidades de las mujeres con discapacidades deben o no incorporarse a los documentos y políticas de las mujeres en general. Sobre este 
debate, Sanjosé (2007), plantea que aunque lo que se busque sea eliminar la discriminación en el goce de los derechos reconocidos a todas las personas, los documentos específicos para la población con discapacidad establecen la necesidad de adoptar medidas específicas para evitar la discriminación. Por su parte, Vélez (2008) se posiciona en el planteamiento de que las personas que pertenecen a grupos marginados tienen los mismos derechos humanos que los demás y que "en ningún lugar de la Declaración de los Derechos Humanos se indica que los derechos que ahí se otorgan están condicionados a que la persona sea considerada apta para ejercerlos" (p. 11).

Como estrategia para ir armonizando estas posturas, Muñiz (2006), nos presenta la postura de 'la igualdad en la diferencia' como respuesta al 'dilema de la diferencia'. Este autor indica que "el dilema de la diferencia plantea la tensión entre dos alternativas que la misma autoridad estatal puede adoptar al tener que hacer frente a las diferencias" (p. 113). De un lado, se puede decidir aplicar un trato igual, sin tomar en consideración las diferencias y esto resultar en un discrimen. Por otra parte, se puede incorporar la diferencia y justificar un trato especial, lo que puede llevar a una situación de exclusión. Ante estas situaciones Scott (según citado por Muñiz, 2006) propone la postura de la igualdad en la diferencia. Desde esta perspectiva la igualdad es pensada a partir de la diferencia. La equidad entre los seres humanos significa alcanzar la igualdad en el reconocimiento de la diferencia, lo que amerita cambiar pautas sociales, jurídicas, educativas, etc. En mi opinión desarrollar esta perspectiva e incorporarla en los procesos de políticas públicas tendría un impacto positivo en la vida de las mujeres con discapacidades, pues ayudaría a deconstruir la postura de que la diferencia es sinónimo de desigualdad y de inferioridad.

Otras estrategias identificadas para lidiar con la discriminación que sufren las mujeres con discapacidades en los asuntos de política pública y de servicios la ofrece el Plan de Acción para las Mujeres con Discapacidad (2007), en España. En este documento se plantea que la situación de discrimen y marginalidad que enfrentan las mujeres con discapacidades es tan seria, profunda y estructural que requiere "aunar la oportunidad de las políticas de transversalidad y las políticas específicas" (p. 3). Las políticas de transversalidad buscan 
introducir la perspectiva de discapacidad en las políticas de género y las políticas específicas son medidas de acción positiva dirigidas a reducir la brecha entre hombres y mujeres.

Queda claro que existen diferentes posturas y perspectivas sobre si las necesidades e intereses de las mujeres con discapacidades físicas deben o no estar incluidas en las políticas o documentos internacionales que atienden los asuntos del género femenino y que también se han desarrollado estrategias que pueden ayudar a conciliar dichas posturas. En mi opinión, el reconocimiento de las diferencias es fundamental en el proceso de visibilizar a las mujeres con discapacidades físicas como parte del colectivo femenino. Esto no implica degradar su estatus ciudadano, busca que se atiendan sus necesidades e intereses particulares. Las estrategias que se utilicen ya sea de transversalidad, desarrollando protocolos específicos dentro de las políticas generales o creando políticas específicas, va a depender de la situación y el contexto social y político en el que se esté generando el análisis.

En Puerto Rico, no existen políticas públicas que tengan como sujeto principal a las mujeres con discapacidades y estas son invisibles a los ojos de quienes crean políticas públicas y programas para mujeres. Esta realidad abre un amplio camino para la investigación, la formulación e implantación de políticas públicas, así como para el desarrollo de programas desde paradigmas diferentes. Estos nuevos paradigmas permitirán romper con las posturas dominantes que niegan las diferencias y que promueven el mantenimiento de visiones negativas, opresoras y discriminatorias contra las mujeres con discapacidades físicas. Analizar e introducir las estrategias que se identifican en este escrito, podría ser un comienzo.

\section{Reflexiones finales}

No cabe duda de que las mujeres con discapacidades físicas son sujetos de derechos. Así está consignado en la Constitución de nuestro país y en documentos internacionales. Sin embargo, no podemos negar que esa inclusión tácita no ha promovido la visibilización de este sector poblacional en asuntos de políticas públicas y de servicios. Entonces, ¿son necesarios los derechos especiales para las mujeres con discapacidades físicas? Para responder a esta pregunta acojo las siguientes palabras: "no se trata de fragmentar los derechos o de buscar derechos especiales, lo necesario es darle la más 
amplia significación a los derechos humanos, al ejercicio de la sexualidad" (Mogollón, s. f., párr.. 7). Nuestra realidad política, social y religiosa es compleja y excluyente para amplios sectores poblacionales. Este es uno de los retos que enfrentamos hoy las personas que ejercemos la profesión del Trabajo Social.

\section{Para la academia}

Quienes ejercemos el Trabajo Social solemos tener una visión estrecha en cuanto al alcance de nuestra profesión. Es por esto que no establecemos como objetivos de nuestras intervenciones que quienes participen de nuestros servicios logren una mayor consciencia sobre su situación individual y colectiva, sobre las estructuras sociales que los oprimen y que actúen sobre ellas para transformarlas. Aún así, cuando se trata de asumir la práctica indirecta del Trabajo Social, que incluye la administración, adiestramientos, planificación y análisis de políticas públicas, solemos demostrar miedos, inseguridades y negamos tener conocimientos en esas áreas. Seda (según citada por Guardiola, 1998), señala la responsabilidad que tiene la academia en este asunto, al indicar que la educación en Trabajo Social está dirigida a formar profesionales con poca visión respecto a la responsabilidad que deben tener como formuladores, analistas y evaluadores de política social. La carencia de educación formal en las áreas de servicio indirecto y el enfocarse en el individuo y no en las estructuras sociales, económicas, políticas y religiosas que lo impactan ha tenido consecuencias para un enfoque integral del Trabajo Social. Es importante reconocer, sin embargo, que esta es una realidad que va cambiando para mejorar. Un ejemplo de esto es la decisión tomada por el personal de la Escuela Graduada de Trabajo Social de la Universidad de Puerto Rico, Recinto de Río Piedras de crear y desarrollar un programa doctoral dirigido al análisis de políticas públicas y a la administración. A esto se añade el impacto que están teniendo las personas egresadas de dicho programa doctoral en las diferentes universidades del país. Estos cambios en la academia, que lleva a que más trabajadores/as sociales se eduquen formalmente en el análisis de las políticas públicas y en la administración,tiene un efecto multiplicador lo que permite que más estudiantes de Trabajo Social valoren éstas áreas del quehacer profesional. 


\section{Para quienes hacen políticas públicas y profesionales}

No cabe duda de que, en el caso que nos ocupa, hay mucho que hacer desde el Trabajo Social. Para ello, se requiere una visión amplia, nacional y libre de prejuicios para concienciar a la sociedad y a quienes hacen políticas públicas de la necesidad de incorporar las voces de las mujeres con discapacidades físicas en todos los temas de la vida pública que les atañen, incluyendo el de la sexualidad. Realizar este trabajo requiere de la identificación, educación y apoderamiento de estas mujeres para que conozcan cuáles son sus derechos y las formas de exigirlos. Definitivamente la realidad que enfrentan las mujeres con discapacidades físicas para ejercer sus derechos, específicamente los derechos sexuales y reproductivos, requiere de profesionales del Trabajo Social con destrezas en servicio directo e indirecto. Sin embargo, lo más importante será tener consciencia. Dussel (1998) plantea que es necesario tener consciencia para reconocer a cada participante como un sujeto ético distinto. "Ese respeto y reconocimiento del otro es el momento ético originario por excelencia, ya que da lugar al otro para que intervenga en la argumentación no sólo como igual, con derechos, sino como libre..." (Dussel, 1998 , p. 414). Hacia esa liberación es que debe ir dirigida la intervención desde el Trabajo Social. Sin embargo, esta es una responsabilidad que tiene que ser compartida y asumida por el Estado, pues es este el que establece las políticas públicas que incluyen o excluyen a los sectores poblacionales marginales de Puerto Rico.

\section{Para quienes ejercemos el Trabajo Social como profesión}

Planteo la necesidad de que continuemos desarrollando destrezas y creando espacios para la formulación e implementación de políticas públicas nacionales que atiendan de manera responsable y adecuada las necesidades de las poblaciones vulnerables de nuestra sociedad. Dada nuestra relación colonial con los Estados Unidos, el gobierno de Puerto Rico no toma todas las decisiones de los asuntos que impactan a la población. Esto trae consigo el implementar políticas creadas en el extranjero y asumir restricciones impuestas por el gobierno federal para garantizar la asignación de fondos. Un ejemplo de esto son los programas bajo el Título $X$. A través de estos se ofrecen servicios gratuitos a la comunidad en las 
áreas de salud sexual y reproductiva, pero no pueden ofrecerse servicios relacionados con el aborto dadas las restricciones impuestas por quienes le asignan fondos (Otón, 2010). Esto por mencionar sólo un caso.

De todas maneras, como trabajadora social, como mujer que también he sido identificada como esa otra y como puertorriqueña reconozco la necesidad de trabajar para que se logre la incorporación de las voces de las mujeres con discapacidades físicas en los procesos públicos que impactan sus vidas como mujeres y como mujeres con discapacidades. Lograr la amplitud de las políticas públicas que atienden a las mujeres en general y la creación de políticas donde, de manera transversal, se incluyan las perspectivas de género y de discapacidad, son puntos de partida, así como aprovechar los espacios públicos para presentar y problematizar sobre el tema. Eroles (2002) menciona que "uno de los mayores retos del presente es lograr que la construcción de la ciudadanía sea tan amplia que incluya a la totalidad de las personas de una comunidad democrática" (p. 24). Este reto social, político y profesional no puede seguir siendo postergado, es hora de asumirlo. 


\section{Referencias}

Anastasiu, Dimitris, \& Kauffman, James M. (2011). A social constructionist approach to disability: Implications for special educations. Exceptional Children, 77(3),367-384.

Barton, Len. (1998). Discapacidad y sociedad. Madrid: Ediciones Morata.

Becker, Heather, Stuifberguen, Alexa, \& Tinkle, Mindy. (1997). Reproductive health care experiences of women with physical disabilities: A quality study. Archives of Physical Medicine and Rehabilitation, 5, 26-33.

Braddock, David L., \& Parish, Susan L. (2001). An institutional history of disability. En Gerd Albrecht, Katherine. Seelman \& Michael Bury (Eds.), Handbook of disability studies (pp. 11-54).

Cruz Pérez, María del Pilar. (2004, otoño). Mujeres con discapacidad y su derecho a la sexualidad. En Política y Cultura. Recuperado de http://www.xoc.uam.mx/ polculpyc22/07-cruz.pdf .

Dussel, Enrique. (1998). Ética de la liberación en la edad de la globalización y de la exclusión. Madrid: Editorial Trotta.

Eroles, Carlos. (2002). La discapacidad como eje de un movimiento social de afirmación de derechos. En Carlos Eroles \& Carlos Ferreres (Eds.), La discapacidad: Una cuestión de derechos humanos (pp. 15-39).

Esmail, Shaniff, Darry, Kim, Walter, Ashlea, \& Knupp, Heidi. (2010). Attitudes and perceptions towards disability and sexuality [Electronic version]. Disability and Rehabilitation,32(14), 1148-1155.

European Disability Forum. (2010). Contribución del EDF al debate general del CESCR sobre los derechos sexuales y reproductivos. Recuperado de http://www.edf-feph.org

Federación Internacional de Planificación Familiar. (1995). Carta de los Derechos Sexuales y Reproductivos (IPPF). Recuperado de http://www.cosladajoven.net/

Federación Internacional de Planificación Familiar. (2008). Derechos Sexuales: Una Declaración de la IPFF. Recuperado de http:// www.ippf.org

Flórez, Carmen E., \& Soto, Victoria E. (2008). El estado de la salud sexual y reproductiva en América Latina y el Caribe: Una visión global. Banco Interamericano del Desarrollo. Recuperado de http://www.aibd.org/res/publications/

Gil Ruiz, Sol B., Rodríguez Montes, María del Carmen, \& Soto Caldero, Joharelis. (2010). La imagen corporal, la estima sexual y la satisfacción de las relaciones en un grupo de personas 
con Distrofia Muscular. Disertación doctoral no publicada, Universidad de Puerto Rico.

Irish Family Planning Association. (s. f.). Disability and sexuality briefing report. Recuperado de http://ifpa.ie/media-Infol

Las Normas Uniformes sobre Igualdad de Oportunidades para las Personas con Discapacidad. (2002). En Carlos Eroles \& Carlos Ferreres (Eds.), La discapacidad: Una cuestión de derechos humanos (pp. 103-127). Buenos Aires: Editorial Espacio.

López González, María. (2007). Discapacidad y Género. Estudio Etnográfico sobre mujeres discapacitadas. Educación y Diversidad: Revista Interuniversitaria de investigaciones sobre discapacidad e interculturalidad. Recuperado de http:// www.dialnet.unirioja.es/servlet/

Manifiesto de las Mujeres con Discapacidad. (2004). Recuperado de http://www.canfcocemfe.org/programas/mujer/manif-mujer_04. pdf

Ministerio de Trabajo y Asuntos Sociales de España. (2006). Plan de acción para las mujeres con discapacidad 2007. Recuperado de http://www.uab.cat/Document/

Merali, Isfahan. (2002). Advancing women's reproductive and sexual health rights: using the International Human Rights system. Development in Practice, 10(5), 609-624. Doi: 10.1080/09614520020008797.

Mogollón, María Esther. (s.f.). Cuerpos diferentes - Sexualidad y reproducción en mujeres con discapacidad. Recuperado de http://www.centreantigona.uab.cat/docs/

Montañez Concepción, Isabel. (2010). El escolar con discapacidad y su familia: Intervención social que los apodera. En Jorge Benítez \& Astrid Santiago (Eds.), Ciudadanía y exclusión en Puerto Rico (pp. 81-103). San Juan, P. R.: Editorial Tal Cual.

Morris, Jenny. (Ed.) (1996). Encuentros con Desconocidas: feminismo y discapacidad. Madrid: NARCEA Ediciones.

Muñiz Fernández, Manuel E. (2006). Menores con discapacidad en Puerto Rico: Problemática sociojurídica de la discapacidad y límites del derecho. San Juan: Editorial Publicaciones Puertorriqueñas.

Nosek, Margaret A., Rintala, Diana, Young, Marie E., Howland, Carol A. et al. (1997). Sexuality issues among women with physical disabilities. Journal of Rehabilitation Research and Development, 34, 244-245. Recuperado de ProQuest Medical Library. (Documento ID: 11684570). 
Oliver, Mike. (1998). Capítulo II: ¿Una sociología de la discapacidad o una sociología discapacitada? En Len Barton, Discapacidad y Sociedad. Madrid: Ediciones Morata.

Otón, Patricia. (2010). Agenda inconclusa: Derechos sexuales y reproductivos desde las la perspectiva de salud. Revista Jurídica UPR, 79(3), 851-877.

Parodi, Luis M. (2003). La educación especial y sus servicios. Río Piedras: Publicaciones Puertorriqueñas.

Samperio, Elsa. (2004). Inclusión y exclusión, aspectos de una misma realidad. Contribución al estudio de la problemática de la discapacidad.

Sanjosé Gil, Amparo. (2007). El primer tratado de derechos humanos del siglo XXI: la Convención sobre los Derechos de las Personas con Discapacidad (Versión Electrónica). Revista Electrónica de Estudios Internacionales, 13, 1-26. Recuperado de http:// www.reei.org/reei\%2013/SanjoseGil(reei13).pdf

Soler, A. Teixeira, \& Jaime, V. (2008). Discapacidad y dependencia: Una perspectiva de género. XI Jornada de Economía Crítica. Recuperado de http://www.uc.es/info.ec/

U.S. Census Bureau, Census 2000 Summary File 3.

Vargas Pelayo, Claudia S. (2007). El significado de los derechos sexuales y reproductivos de las mujeres con discapacidad en Autlán Jalisco. Recuperado de http://educacionjalisco.gob. $\mathrm{mx} /$ ciielpdf/congreso2007/vulnerables/ponencia

Vélez, Carmen Milagros. (2007). Construcciones sociales en torno a las experiencias de discapacidad desde las voces de mujeres de los cuerpos diferentes, de frente a voces de las políticas públicas. Disertación doctoral no publicada, Universidad de Puerto Rico, Río Piedras.

Vélez, Carmen Milagros. (2008). Ciudadanía especial: Un análisis de la Carta de Derechos de las Personas con Impedimentos. Revista Análisis, IX, 1 -17.

Villalba Rolón, Lorenzo. (2007, junio). Resolución Concurrente del Senado 81. Ponencia inédita presentada ante la Comisión de Gobierno y Asuntos Laborales del Senado de Puerto Rico en San Juan, PR.

Viñuela Suárez, Laura. (2009). Mujeres con discapacidad: Un reto para la teoría feminista. Feminismo/s, 13, 33-48. Recuperado de http://www.rua.ua.es/dspace/bitstream/ 